\title{
Articulación de las competencias específicas de las asignaturas del área de Filosofía Práctica, con el perfil de egreso de la Licenciatura en Filosofía, Ética y Valores.
}

\begin{tabular}{|c|c|}
\hline Pág. 144-168 & Resumen \\
\hline $\begin{array}{l}\text { Anibal Beitía } \\
\text { A. }\end{array}$ & $\begin{array}{l}\text { El estudio de carácter descriptivo-analítico para contrastar las } \\
\text { competencias específicas de las asignaturas del área de Filosofía Práctica } \\
\text { con el perfil de egreso, busca evidenciar la articulación de las } \\
\text { competencias específicas o profesionales. De igual forma, pretende } \\
\text { explicar, a través de encuestas aplicadas a estudiantes y profesores, las }\end{array}$ \\
\hline $\begin{array}{l}\text { Universidad de } \\
\text { Panamá } \\
\text { Facultad de } \\
\text { Humanidades }\end{array}$ & $\begin{array}{l}\text { estrategias didácticas utilizadas durante el proceso formativo, } \\
\text { conducentes a la apropiación de competencias fundamentales para el } \\
\text { ejercicio profesional del quehacer filosófico. }\end{array}$ \\
\hline anibal.beitiaup.ac.pa & $\begin{array}{l}\text { Los resultados del estudio, identifican como hallazgo significativo que es } \\
\text { necesario reforzar la malla curricular de la Licenciatura en Humanidades } \\
\text { con especialización en Filosofía, Ética y Valores. Por otra parte, se } \\
\text { evidencia también la necesidad de promover el uso de métodos y } \\
\text { estrategias para la enseñanza y el aprendizaje de la Filosofía, tendientes a } \\
\text { potenciar el nivel de apropiación de las competencias específicas - } \\
\text { profesionales del futuro Licenciado en Filosofía. }\end{array}$ \\
\hline $\begin{array}{l}\text { Fecha de entrega: } \\
\text { agosto, } 2020\end{array}$ & $\begin{array}{l}\text { Palabras clave: Articulación, aprendizaje, competencia, competencia } \\
\text { específica, diseño curricular, filosofía práctica, perfil de egreso }\end{array}$ \\
\hline $\begin{array}{l}\text { Fecha de } \\
\text { aceptación: } \\
\text { noviembre, } 2020\end{array}$ & \\
\hline
\end{tabular}




\title{
Articulation of the specific competences of the subjects in the area of Practical Philosophy, with the graduate profile of the degree in Philosophy, Ethics and Values
}

\begin{abstract}
The descriptive-analytical study to contrast the specific competences of the subjects in the area of Practical Philosophy with the graduate profile, seeks to demonstrate the articulation of specific or professional competencies. Similarly, it aims to explain, through surveys applied to students and teachers, the didactic strategies used during the training process, leading to the appropriation of fundamental competencies for the professional exercise of philosophical work.

The results of the study identify as a significant finding, that it is necessary to reinforce the curriculum of the Bachelor of Humanities with a specialization in Philosophy, Ethics and Values. On the other hand, there is also evidence of the need to promote the use of methods and strategies for teaching and learning Philosophy, aimed at enhancing the level of appropriation of specific competences - professionals of the future Graduate in Philosophy.
\end{abstract}

Keywords: Articulation, learning, competence, specific competence, curriculum design, practical philosophy, graduation profile. 


\section{INTRODUCCIÓN}

La Educación Superior evoluciona hacia un modelo en que profesores y estudiantes serán, ante todo, aprendices permanentes y en el que los programas de estudio se difundirán en función de los nuevos conocimientos y las nuevas tecnologías de enseñanza y aprendizaje. Así, se hace necesario que la formación filosófica universitaria tenga también un Plan de Estudio coherente con las necesidades de nuestros tiempos. Flamarique (2008), señala que: "la filosofía no puede quedarse impasible ante el horizonte que dibujan las últimas reformas universitarias: la articulación de los saberes ha sido competencia de la filosofía desde sus inicios” (p.98).

Al respecto, Solange, Herrero y García (2007), reconocen que es necesario "reconstruir el debate actual en torno al estatuto de la actividad filosófica, así como indagar las formas de articulación entre discurso filosófico, práctica educativa, y las formas de racionalidad involucradas en las mismas" (p.159).

El currículum ha sido definido en tanto sistema, visualizándose como una realidad compleja y dinámica en la que un conjunto de elementos o unidades interactúan a través de distintos procesos para lograr la formación integral del educando. (Santiváñez, 2012).

Tovar y Sarmiento (2011), afirman que en la actualidad se "exige interpretar el currículo como proceso ampliamente temporalizado y de construcción/deconstrucción por parte de los agentes involucrados, y no como un programa limitado en su realización por unos objetivos y logros predeterminados e inmodificables" (p.509).

El "currículum pensado", entendido como la planificación estructurada del proyecto educativo, es para algunos autores el diseño curricular, por cuanto busca articular y garantizar la formación integral (académica, tecnológica y humanística) de las personas. Al respecto, Ortiz (2014), señala que es el "plan que se concibe, en el que queda reflejado el enfoque educativo que se desea" (p.43).

En general, el diseño curricular incluye, acciones metodológicas, aspectos de contenidos temáticos, estrategias evaluativas y la caracterización del tipo de persona que se desea formar o preparar según el contexto sociocultural y la época que se vive. Entendido como sistema, el 


\section{Anibal Beitía A.}

diseño curricular, considera cinco elementos fundamentales: perfil, objetivos, contenido, estrategias didácticas y evaluación.

Los principales componentes del diseño curricular se agrupan en dos grandes apartados relacionados entre sí:

- Primer apartado: incluye la justificación, la fundamentación, el perfil del egresado y los objetivos generales de la carrera.

En cuanto al perfil del egresado, éste describe y justifica las competencias generales y específicas que ha de cultivar el nuevo profesional, al concluir el proceso de formación académica. Se refiere, en consecuencia, al conjunto estructurado de capacidades y atributos que la Universidad se compromete a cultivar en el futuro profesional, de modo que pueda insertarse con éxito en el mundo laboral. De ahí que, en el Plan de Estudio, aparece frecuentemente el perfil genérico y el perfil específico de la carrera. El perfil genérico se refiere a aquellas competencias básicas para la convivencia humana $y$, en consecuencia, son comunes a diversas profesiones. El perfil específico lo constituyen aquellas competencias muy propias de cada profesión en concreto. Estas capacidades, habilidades y acciones, necesitan un nivel de mayor exigencia formativa tanto a nivel teórico, como práctico (Santiváñez, 2012).

- Segundo apartado: se presenta la estructura curricular, que incluye entre otros aspectos, la malla curricular, la organización del contenido curricular, la descripción de los cursos con sus respectivos créditos, la agrupación de conocimientos por áreas, entre otros.

La estructura curricular: Está constituida por una relación intrínseca entre los principales elementos del diseño curricular. Tiene como finalidad ofrecer lineamientos pedagógicos, tecnológicos y prácticos en el proceso formativo de los futuros egresados de una carrera. Aquí se toman en cuenta tanto las áreas disciplinarias como los ejes curriculares. Para que la estructura curricular mantenga coherencia interna, se ha de tener claridad en los criterios de selección y 
organización del contenido curricular, la cantidad de créditos para cada asignatura, los requisitos de ingreso y de egreso, nombre del título que se ofrece, campo ocupacional del futuro profesional, entre otros. La estructura de la malla curricular ha de contemplar a su vez las diversas unidades de aprendizaje con sus respectivas experiencias formativas. Pues es precisamente la malla curricular ese "conjunto de espacios formativos articulados entre sí mediante los cuales, se busca logren el perfil de egreso en un programa de formación” (Tobón, 2013, p.185).

\section{El currículum desde un enfoque basado en competencias.}

La definición más frecuente para el concepto competencia, es el de "saber hacer desde un contexto". El Proyecto Tuning (2006), considera las competencias como un conjunto de capacidades cognitivas y metacognitivas; capacidades a su vez, intelectuales, prácticas y éticas; donde la prioridad es el aprendizaje del estudiante y el docente se convierte en un diseñador de espacios de aprendizaje a través de un adecuado proceso de planeación didáctica.

Desde esta perspectiva, González (2007), hace referencia a la competencia como un saber hacer con conciencia; un saber que se convierte en acción. Es decir, un saber cuyo sentido inmediato no sería tanto el de "describir" la realidad, sino más bien de transformarla; no se trata tanto del saber definir problemas, sino más bien del aprender a solucionarlos eficazmente.

Por otra parte, Tobón (2013), afirma la "necesidad de articular los saberes de distintas disciplinas, con base en actividades inter y transdisciplinarias que lleven a la superación del modelo tradicional de asignaturas" (p.59). Todo lo cual indica que las competencias contienen aspectos que se adquieren a través de la práctica interactiva desde un contexto sociocultural-educativo. Las mismas se van desarrollando en la medida en que se van incorporando nuevas alternativas $u$ opciones de acción oportuna para resolver situaciones en creciente grado de complejidad. A través de las competencias, la persona va haciendo ajustes en sus procesos cognitivos y los va contextualizando.

Generalmente, las competencias se clasifican en tres grandes grupos: las básicas (asociadas a conocimientos fundamentales tales como habilidad para la lectoescritura, etc.); las genéricas 


\section{Anibal Beitía A.}

(hacen referencia a actitudes axiológicas prácticas, que permite integrar diversos saberes, como trabajar colaborativamente, etc.) y las específicas (se refieren a aspectos concretos que una determinada profesión requiere). Estas últimas, se refieren a las capacidades indispensables que el alumno debe adquirir y desarrollar para asegurar el ejercicio de determinada profesión.

Por ello, se requiere de una acertada articulación entre las competencias específicas, que se han de trabajar durante el proceso de enseñanza-aprendizaje de cada asignatura del Plan de Estudio, y el perfil de egreso de la carrera universitaria. El enfoque curricular por competencias articula de modo pertinente, contenidos, recursos y metodologías en la formación de los futuros profesionales que la sociedad demanda.

En la actualidad, todo diseño curricular ha de elaborarse seleccionando de modo muy reflexivo los contenidos de aprendizajes cognitivos, procedimentales y actitudinales que favorezcan el crecimiento académico, humanístico y científico de aquel profesional egresado de una institución educativa superior.

El aprendizaje por competencia, se inserta en la política educativa a nivel mundial. De esta forma, se van posicionando una gran variedad de "cambios educativos por la introducción del aprendizaje autónomo, el aprendizaje significativo, el constructivismo, la metacognición y el surgimiento de nuevas teorías de la inteligencia" (Tobón, 2013, p.57).

\section{Competencias para la formación del profesional del quehacer filosófico.}

A continuación, nos referiremos a algunas competencias que consideramos indispensables para la formación profesional en filosofía.

- Visión de interdisciplinariedad, esto con el fin de suscitar reflexiones filosóficas que tomen en cuenta otros saberes. Esto exige, entre otras cosas, participación de encuentros con profesionales de otras disciplinas para el desarrollo de habilidad del "trabajo colaborativo", investigaciones, proyectos. 
- Capacidad crítico-creativa, que permita el abordaje con profundidad y seguridad en nuevos campos, como la Neurociencia, Bioética, Biotecnología, entre otros. Esta competencia del pensamiento creativo permite "generar ideas originales e ingeniosas, combinarlas de una manera nueva y productiva y descubrir asociaciones poco comunes entre ellas" (Sanz de Acedo, 2012, p.69). Todo ello implica aprender a ver la realidad desde diferentes puntos de vista.

- Solución de problemas y toma de decisiones, supone cultivar la metacognición, que implica actuar de manera reflexiva para evaluar conscientemente los resultados obtenidos y hacer los ajustes correspondientes, tanto en situaciones individuales o grupales.

\section{Competencias propias para el área de Filosofía Práctica.}

En el caso de la Filosofía Práctica, entendida como el conjunto de disciplinas filosóficas que estudian la acción humana y sus fines, se profundiza la relación entre el pensamiento y el comportamiento humano y los efectos inevitables de esta acción; implicaría, por consiguiente, aprender a pensar creativa y reflexivamente a partir de los contenidos temáticos de las asignaturas y hacer el contraste con los grandes problemas de la sociedad actual.

En el ejercicio de la tarea filosófica, es imprescindible, desarrollar la capacidad crítico-creativa. Esta competencia permite "generar ideas originales e ingeniosas, combinarlas de una manera nueva y productiva y descubrir asociaciones poco comunes entre ellas" (Sanz de Acedo, 2012, p.69). Todo ello implica aprender a ver la realidad desde diferentes puntos de vista; es decir, apertura a los diversos aportes que desde las otras disciplinas se pueden hacer en el abordaje de un tema o problemática humana.

Desde esta perspectiva, es que "el saber filosófico se enseña en la Universidad bajo forma de métodos de investigación, de categorías, de conceptos, de criterios de validez de argumentación y de estructuras más o menos formales permitiendo construir los mundos físico, histórico, moral y racional" (UNESCO, 2009, p.38). 
Anibal Beitía A.

El currículum por competencias de la Licenciatura en Humanidades con especialización en Filosofía, Ética y Valores de la Universidad de Panamá.

La Universidad de Panamá, como Institución de Educación Superior, tiene entre sus finalidades, la difusión del conocimiento a través de la formación científica, tecnológica, humanística y cultural, desde la perspectiva de la excelencia académica con una actitud crítica y productiva (Estatuto Universitario, Art. 2).

Desde el año 2004, esta Universidad, ha iniciado un proceso de cambios curriculares, para asegurar la formación de los futuros profesionales que demanda la sociedad.

El Modelo curricular adoptado por la Universidad de Panamá, es el de Formación por Competencias (2008), que considera tres áreas de competencia: cognitivas, procedimentales y la interpersonales/valóricas/actitudinales.

- Cognitivas: fundamentadas en conocimientos disciplinares, saber, comprender, analizar.

- Procedimentales: integración de las acciones prácticas, saber cómo proceder en situaciones profesionales determinadas, elaborar proyectos, operar sistemas tecnológicos, físicos, informáticos y sociales.

- Interpersonales/ valóricas/actitudinales: permiten cooperar e interactuar con otros en función de un objetivo común, saber comportarse en situaciones diversas, participar y comprometerse, saber percibir situaciones.

A partir del planteamiento anterior, se consideran tres tipos de competencias:

- Básicas: Esenciales, las más importantes. Implícitas en las prácticas laborales y en la educación. Son las capacidades indispensables para el aprendizaje de una profesión. En ellas se encuentran las competencias cognitivas, técnicas y metodológicas, muchas de las cuales son adquiridas en los niveles educativos previos (lenguaje oral, escrito, matemático). 
- Genéricas: Necesarias para el desempeño de tareas, incluyen las básicas y se vinculan con la comunicación de ideas, manejo de la información, solución de problemas, trabajo en equipo (análisis, planeación interpretación, negociación). Posibilitan las transferencias de competencias laborales por lo que se denominan transversales.

- Específicas o profesionales: típicas de cada uno de los profesionales. Pueden tener relación en proporción importante con aquellas de otras profesiones pertenecientes a la misma familia de ocupaciones.

En la Universidad de Panamá, de acuerdo a la Política Académica Curricular, los Planes de estudio de las carreras constan de 2 áreas: Área de Formación General (que incluye asignaturas culturales y propedéuticas o de iniciación de la disciplina) y que a su vez integran el Núcleo Común de formación; y el Área de Formación Profesional, relacionada directamente con la carrera y que incluye asignaturas de Apoyo (marco de referencia y que actúan como prerequisito) y las Asignaturas Fundamentales o Específicas, propias de la carrera.

En 2014, la Comisión Curricular del Departamento de Filosofía de la Facultad de Humanidades, concreta la actualización de la Licenciatura en Humanidades con especialización en Filosofía, Ética y Valores (en adelante, se utilizará Licenciatura / Licenciado en FEV); la misma posee 140 créditos, 144 horas de clases y 49 asignaturas agregadas como sigue:

\section{Tabla 1}

Estructura del Plan de Estudio de la Licenciatura en FEV, asignaturas según área.

\begin{tabular}{ll}
\multicolumn{1}{c}{ Áreas } & \multicolumn{1}{c}{$\begin{array}{l}\text { Número de } \\
\text { asignaturas }\end{array}$} \\
\hline Área de Formación General & 7 \\
Área de Formación Profesional. & 42 \\
Apoyo & 23 \\
*Específicas & 19 \\
Total & 49 \\
\hline
\end{tabular}

*Asignaturas del área de Filosofía Práctica- 45.23\% de formación ética-axiológica. 


\section{Anibal Beitía A.}

Las asignaturas del área de formación profesional (correspondientes al área de Filosofía Práctica), son las siguientes: Axiología I, Axiología II, Ética I, Ética II, Antropología Filosófica, Filosofía de la Educación, Filosofía de la Religión, Filosofía del Derecho, Filosofía de los Derechos Humanos I, Filosofía de los Derechos Humanos II, Religiones Comparadas, Teoría del Estado y Constitución, Fenomenología de la Religión, Bioética I, Bioética II, Filosofía Política, Filosofía Social, Deontología y Teorías Emergentes.

\section{EI Perfil de Egreso de la Licenciatura en FEV de la Universidad de Panamá.}

El diseño curricular de la carrera de Licenciatura en FEV, se actualizó en 2014 y presenta el perfil académico-profesional del egresado en tres áreas, a saber: de conocimiento, de habilidades y de actitudes.

A modo de síntesis, el egresado como Licenciado en FEV, posee un profundo conocimiento de las disciplinas filosóficas más relevantes y del desarrollo histórico de la filosofía; aplica las teorías y corrientes filosóficas en la interpretación de los diferentes aspectos de la cultura actual; emplea diversos métodos lógicos tanto para la enseñanza de la filosofía como para la solución de problemas sociales y humanos que afectan a la Comunidad; manifestando así, una actitud reflexiva, de apertura al diálogo y tolerancia con pensamientos diferentes. En la Tabla 2, están los 22 aspectos (separados por áreas), que conforman el Perfil de Egreso del Licenciado en FEV.

\section{METODOLOGÍA}

El estudio se enfoca en las asignaturas del área de formación profesional denominada Filosofía Práctica. Aquí es importante señalar que son diecinueve asignaturas del área de Filosofía Práctica, pero no se encontró el Programa Analítico de una de ellas. Por esta razón, es que el estudio se hace con los programas de dieciocho asignaturas. 
La metodología es descriptiva, dado que busca detallar la integración de las competencias específicas contempladas en los programas analíticos de las asignaturas del área de Filosofía Práctica, respecto a las tres áreas (conocimiento, habilidades y actitudes) que describen el perfil académico y profesional.

El estudio es demás explicativo, pues analiza el nivel de relación o articulación existente entre aspectos constitutivos del perfil de egreso con aquellos procesos formativos en el desarrollo de las asignaturas del área de Filosofía Práctica para explicar su significado dentro de los fundamentos del currículum y el modelo pedagógico existente.

Para el análisis curricular, se utilizaron tres matrices, una para cada área que contempla el perfil de egreso (área de conocimiento, área de habilidades y área de actitudes), las que permitieron establecer relaciones respecto a las competencias específicas de las 18 asignaturas del área de Filosofía Práctica que se analizaron.

Adicional, se elaboraron dos cuestionarios de 14 y 8 preguntas, para ser aplicados a estudiantes y docentes respectivamente. Los mismos indagaron sobre el nivel de apropiación que tienen los estudiantes de las competencias específicas, en las asignaturas del área de Filosofía Práctica y sobre las estrategias didácticas desarrolladas en clase. Ambos instrumentos fueron validados por juicio de expertos utilizando la $\mathrm{V}$ de Aiken.

La población en estudio está conformada por:

- Estudiantes: veintiún estudiantes que cursan la carrera de Filosofía, Ética y Valores, distribuidos por años académicos de la siguiente forma: cinco de 1er año (todos con siete asignaturas aprobadas del área de Filosofía Práctica), tres de 2do. año, dos de 3er. año y 11 de 4to. año (que han terminado el Plan de Estudio y están en Seminario como opción de Grado o están "recogiendo” materias).

- Profesores: se consideró una muestra de ocho, que imparten asignaturas del área de Filosofía Práctica. 
Anibal Beitía A.

\section{RESULTADOS}

\section{Análisis de los programas: vinculación de las áreas de competencias, respecto al perfil de egreso.}

Se revisaron los programas de 18 asignaturas, procurando analizar en cada uno, la vinculación de cada área de competencia (cognitivas, procedimentales y actitudinales), respecto al perfil de egreso. Los aspectos a evaluar se detallan en la tabla 2.

\section{Tabla 2.}

Aspectos a evaluar por área de competencia

\begin{tabular}{ll}
\hline Área de & Aspectos a evaluar del perfil \\
\hline Competencia & 1. Posee un profundo conocimiento de las disciplinas filosóficas más relevantes. \\
2.Conoce el desarrollo de la historia de la filosofía. \\
3. Conoce las propuestas metodológicas contemporáneas para la investigación \\
filosófica. \\
4. Conoce las bases conceptuales y metodológicas para la enseñanza de la \\
filosofía, ética y valores. \\
5. Maneja teorías científicas y humanísticas para la solución de problemas \\
filosóficos actuales. \\
1. Analiza y sintetiza información relacionada con los problemas filosóficos. \\
2. Interpreta información de acuerdo con los criterios lógicos de coherencia, \\
consistencia, corrección y validez. \\
3. Aplica teorías y corrientes filosóficas al interpretar diversos aspectos de la \\
cultura actual. \\
4. Aplica recursos teóricos y metodológicos para el análisis de argumentos \\
formales e informales. \\
5. Relaciona situaciones, problemas y teorías desde la perspectiva lógica, \\
filosófica y epistemológica. \\
6. Comunica sus ideas de manera clara y coherente en forma oral y escrita. \\
7. Emplea métodos lógicos filosóficos para la solución de problemas. \\
8. Aplica métodos adecuados para la enseñanza de la filosofía, ética y valores. \\
1 cultiva el sentido crítico al desarrollo histórico de la filosofía. \\
2. Manifiesta actitud humanística frente a la vida y la sociedad. \\
3. Refleja actitud reflexiva, comprometida y solidaria ante los problemas \\
humanos. \\
4. Gusto por promover el conocimiento filosófico en todos los ámbitos de la \\
sociedad. \\
5.Colabora con especialistas de otras disciplinas en el tratamiento de problemas. \\
6. Apertura al diálogo y tolerancia con el pensamiento diferente. \\
7. Sensibilidad filosófica ante problemas sociales actuales. \\
8. Compromiso activo con la solución de problemas sociales y humanos. \\
9. Compromiso con los máximos ideales del género humano. \\
Actitudinales
\end{tabular}


Los criterios de análisis son los siguientes:

- No Existe Relación (NER): No se evidencia ningún rasgo de modo explícito con el aspecto del área del perfil de egreso que se analiza.

- Existe Relación Parcial (ERP): Presenta por lo menos de uno a tres elementos correspondientes al área del perfil de egreso que se analiza.

- Existe Relación Total (ERT): Se evidencian rasgos explícitos con el aspecto del área del perfil de egreso que se analiza.

\section{Área de competencia Cognitiva.}

Para esta área se analizaron cinco aspectos del perfil, entre los que figuran: el conocimiento de las disciplinas filosóficas más relevantes; el conocimiento del desarrollo de la historia de la filosofía; el conocimiento de las propuestas metodológicas contemporáneas para la investigación filosófica; el conocimiento de las bases conceptuales y metodológicas para la enseñanza de la filosofía, ética y valores; el manejo de teorías científicas y humanísticas para la solución de problemas filosóficos actuales.

En el primer aspecto "posee un profundo conocimiento de las disciplinas filosóficas", coinciden diez asignaturas: (Antropología Filosófica, Axiología I, Filosofía de los Derechos Humanos I, Bioética I, Bioética II, entre otras). No obstante, sería necesario que todas las asignaturas del área de Filosofía Práctica articulen en su totalidad este aspecto, pues uno de los objetivos de la carrera es precisamente "proporcionar los conocimientos, marcos de referencia, elementos teóricos y metodológicos para comprender el desarrollo de las ideas filosóficas..."

En el segundo aspecto "conoce el desarrollo de la historia de la filosofía..." en cuatro asignaturas (Fil. del Derecho, Deontología, Bioética I y Bioética II), no existe relación de las competencias específicas con el Perfil de Egreso en este aspecto en concreto. El desarrollo histórico de la Filosofía permite ubicar un determinado planteamiento filosófico en el contexto socio-cultural correspondiente. 
Anibal Beitía A.

En el cuarto aspecto "conoce las bases conceptuales y metodológicas para la enseñanza de la filosofía...", hay tres asignaturas (Antropología Filosófica, Filosofía de los Derechos Humanos 1 y Filosofía de los Derechos Humanos 2), en la que no existe relación de las competencias específicas con este aspecto en particular. Desde el contenido temático de cada asignatura filosófica, se ha de suscitar la importancia de potenciar un asertivo y sistemático proceso de enseñanza - aprendizaje de la filosofía. En la actualidad, el proceso de enseñanza-aprendizaje de la Filosofía requiere de nuevas estrategias pedagógicas que permitan la consolidación de un pensamiento crítico-reflexivo. Una de estas nuevas estrategias es el Método Socrático de Oscar Brenifier, inspirado en el conocido método de la mayéutica socrática. Se desarrolla mediante una "reflexión grupal progresiva, a través de preguntas, reformulaciones y objeciones". (UNESCO, 2009, p. 21). Se trata de un diálogo argumentativo "confrontativo" para que, comprendiendo la idea principal del tema, los alumnos con la ayuda del educador se puedan apropiar de la misma.

\section{Figura 1}

Relación de las competencias específicas por asignaturas con el perfil de egreso (Área de competencia cognitiva).

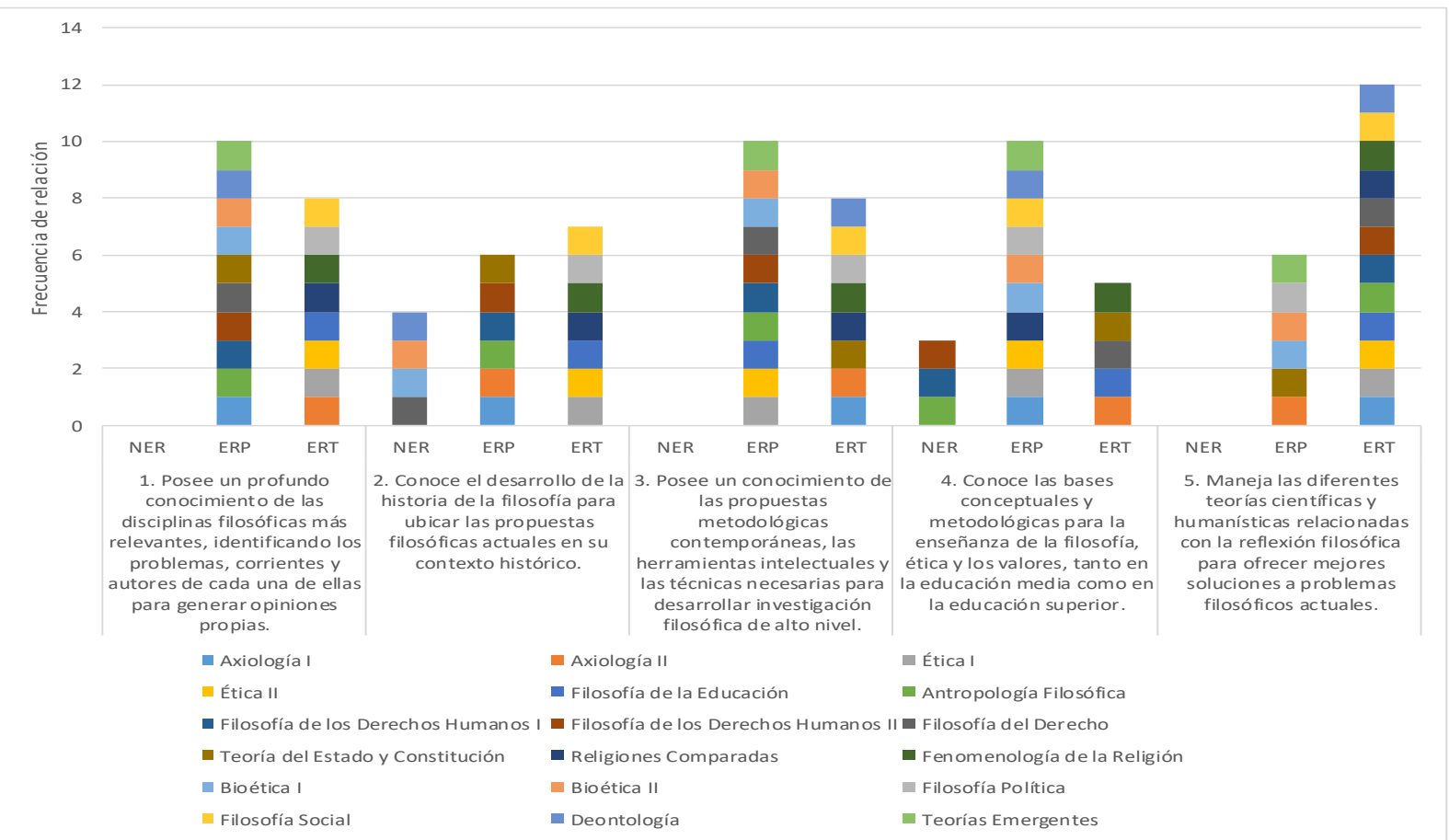

Fuente: Programas analíticos de las asignaturas del área de Filosofía Práctica de la Licenciatura en FEV. 
Llama la atención que, en 12 asignaturas, existe una relación total (ERT), entre las competencias específicas y el quinto aspecto del perfil de egreso ("manejo de teorías científicas y humanísticas para la solución de problemas filosóficos actuales”).

Este hecho lleva a preguntarnos si en el proceso de formación académica del futuro Licenciado en Filosofía, se enseña filosofía o se aprende a filosofar. Objetivamente, pueden ocurrir ambas acciones. El proceso educativo implica tomar en cuenta "...la doble realidad en la que está inmerso: la educabilidad y la educatividad" (Cuéllar, 2012, p.40). La primera consiste en esa capacidad o disponibilidad del ser humano para ser formado, es decir, para ser educado. La segunda, en cambio, se refiere a esa capacidad o facultad del ser humano para educar a otros, para enseñar a "aquél que no sabe eso todavía". Por ello, el proceso de enseñanza lleva consigo la intención de que el otro o los otros, aprendan algo de modo sistemático y significativo.

\section{Área de competencia Procedimental.}

Para esta área se analizaron ocho aspectos, entre los que figuran la habilidad de: analizar y sintetizar información relacionada con los problemas filosóficos; interpretar información de acuerdo con los criterios lógicos de coherencia, consistencia, corrección y validez; aplicar teorías y corrientes filosóficas al interpretar diversos aspectos de la cultura actual; aplicar recursos teóricos y metodológicos para el análisis de argumentos formales e informales; relacionar situaciones, problemas y teorías desde la perspectiva lógica, filosófica y epistemológica; comunicar sus ideas de manera clara y coherente en forma oral y escrita; emplear métodos lógicos filosóficos para la solución de problemas y aplicar métodos adecuados para la enseñanza de la filosofía, ética y valores . 


\section{Figura 2}

Relación de las competencias específicas por asignaturas con el perfil de egreso (Área de competencia procidimental - habilidades).

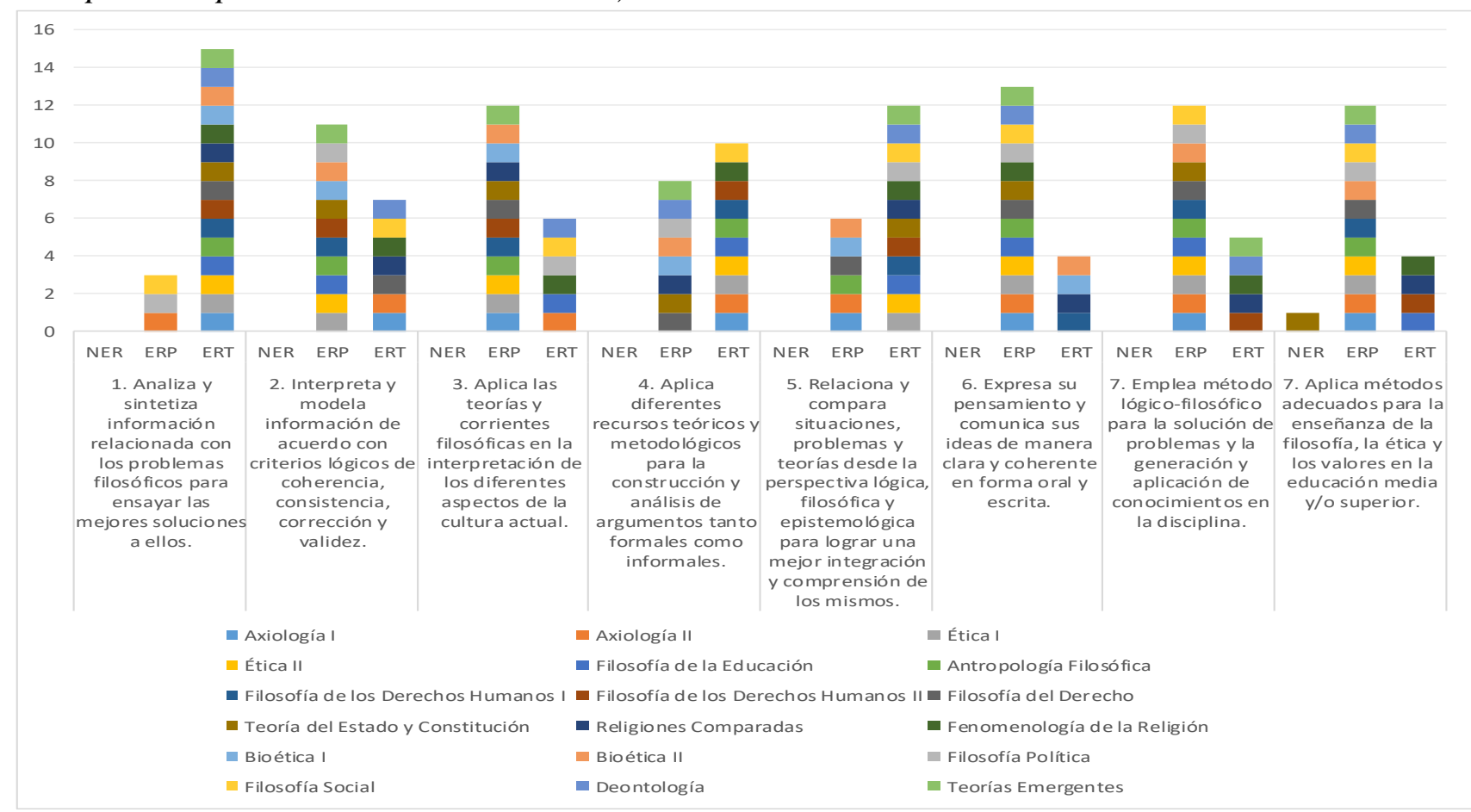

Fuente: Programas analíticos de las asignaturas del área de Filosofía Práctica de la Licenciatura en FEV.

Destaca que en relación al aspecto "Analiza y sintetiza información relacionada con los problemas filosóficos...", la mayor parte de las asignaturas evidencian que Existe relación Total (ERT). Por otra parte, se puede observar que, en seis aspectos, existe una relación parcial (ERP) de las competencias específicas con el perfil de egreso. Estos aspectos son los siguientes: interpreta información con criterios lógicos; aplica teorías y corrientes filosóficas al interpretar diversos aspectos de la cultura actual; aplica recursos teóricos y metodológicos para el análisis de argumentos formales e informales; comunica ideas de manera clara y coherente en forma oral y escrita; emplea métodos lógicos filosóficos para la solución de problemas y, aplica métodos adecuados para la enseñanza de la filosofía, ética y valores.

En el caso de "expresa su pensamiento y comunica sus ideas de manera clara y coherente en forma oral y escrita", trece asignaturas establecen apenas una relación parcial (ERP). 
Para los aspectos tres, siete y ocho (Aplica teorías y corrientes filosóficas al interpretar diversos aspectos de la cultura actual; emplea métodos lógicos filosóficos para la solución de problemas y, aplica métodos adecuados para la enseñanza de la filosofía, ética y valores), hay siete asignaturas que coinciden en establecer una relación parcial. Estas asignaturas son: Filosofía del Derecho, Filosofía del Derecho I, Antropología Filosófica, Ética I, Ética II, Axiología I, Axiología II. Las últimas cinco de estas asignaturas pertenecen al Primer Año de la Licenciatura en FEV: Antropología Filosófica, Ética I, Ética II, Axiología I, Axiología II.

Es clave que el docente fomente competencias específicas desde los inicios de la formación académica universitaria del futuro Licenciado en Filosofía. Esto implicaría la transformación oportuna de las prácticas pedagógicas en el proceso de enseñanza-aprendizaje de la Filosofía. Se trata de cultivar "habilidades" propias para el quehacer filosófico y esto se logra también con adecuadas estrategias didáctico-filosóficas que el docente desarrolle progresivamente.

En el planeamiento didáctico, el docente podría potenciar el logro del aspecto del perfil que indica "la aplicación de métodos adecuados para la enseñanza de la filosofía y la utilización de métodos lógicos para la solución de problemas”. En esa búsqueda de la verdad y en el querer saber el porqué de las cosas, es importante aprender a utilizar, entre otros, el método mayéutico, el método dialéctico, el método racionalista, el método analítico-lingüístico, el método trascendental. Entonces curricularmente, se podría colocar la materia de Métodos Filosóficos en primer año; actualmente está en tercer año.

Lo importante es que la Comisión Curricular al aprobar los Planes de cada carrera, verifique que las competencias específicas estén articuladas con el perfil de egreso y además considere la posibilidad de horas prácticas; actualmente ninguna asignatura de la Licenciatura en FEV, tiene horas prácticas. A través de experiencias de aprendizaje fuera del aula, también se consolida y se construye un nuevo conocimiento científico-filosófico. 
Anibal Beitía A.

Área de competencia Actitudinal.

Para esta área se trabajaron nueve aspectos, entre los que figuran el cultivo del sentido crítico al desarrollo histórico de la filosofía; actitud humanística frente a la vida y la sociedad, actitud reflexiva, comprometida y solidaria ante los problemas humanos; gusto por promover el conocimiento filosófico en todos los ámbitos de la sociedad; colaboración con especialistas de otras disciplinas en el abordaje de problemas; apertura al diálogo y tolerancia con el pensamiento diferente para enriquecer las concepciones del mundo, del hombre y de la sociedad; sensibilidad filosófica ante problemas sociales actuales; compromiso activo con la solución de problemas sociales y humanos y, compromiso con los máximos ideales del género humano.

\section{Figura 3}

Relación de las competencias específicas por asignaturas con el perfil de egreso (Área de competencia Actitudinal).

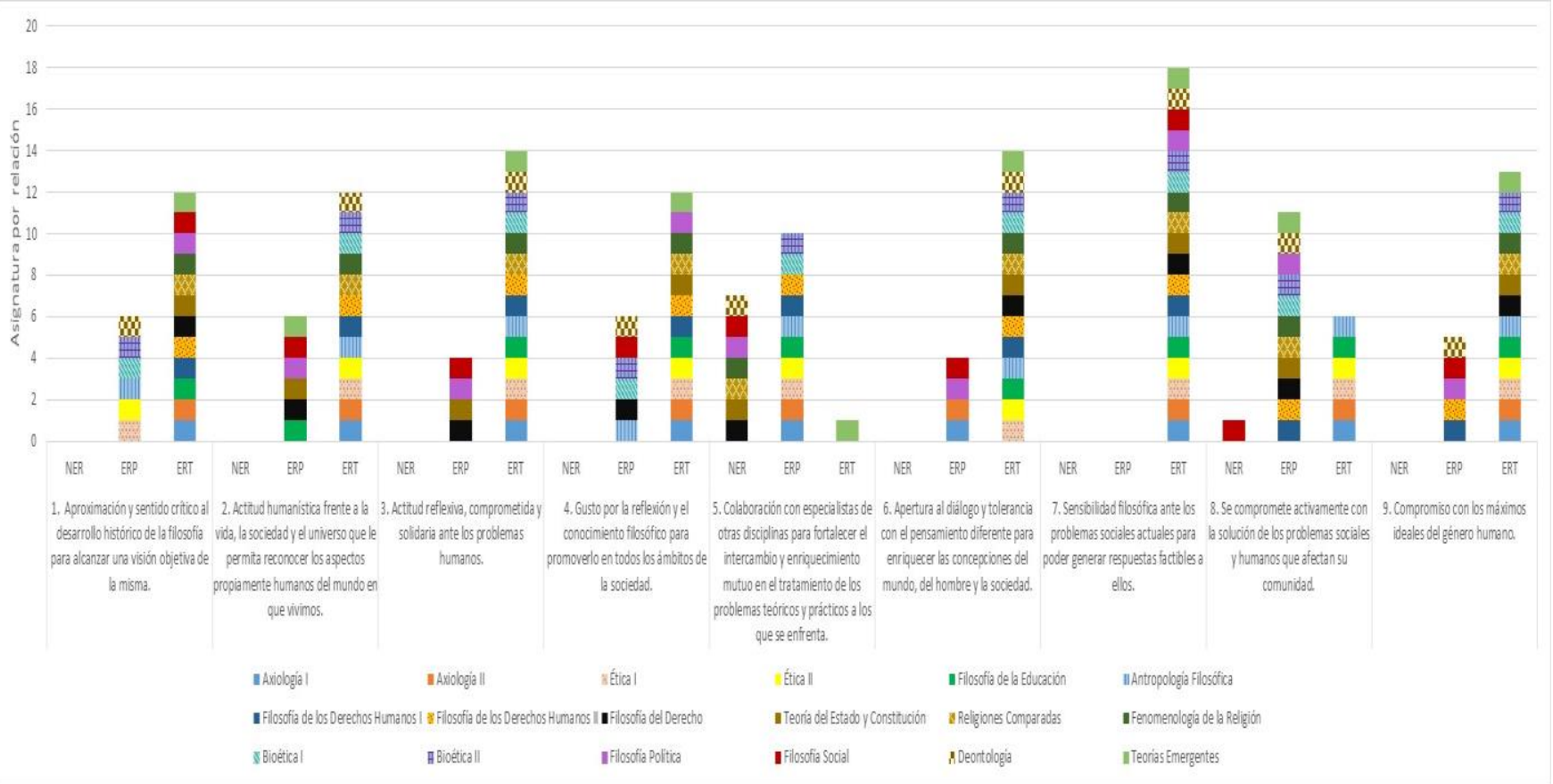

Fuente: Programas analíticos de las asignaturas del área de Filosofía Práctica de la Licenciatura en FEV.

Se observa que, en 7 de 9 aspectos, se indica ERT (existe una relación total) de las competencias específicas con los enunciados del perfil de egreso.

Llama la atención positivamente, que en el séptimo aspecto "sensibilidad filosófica ante los problemas sociales actuales y la actitud reflexiva, comprometida y solidaria ante los problemas 
humanos", todos los programas analíticos presentan relación total (ERT) de las competencias específicas con el perfil de egreso. Esto evidencia que al menos "teóricamente", en los programas analíticos de asignaturas, se visualiza el fomentar una actitud humanista desde la formación académica filosófica.

Sin embargo, es importante señalar que en el quinto aspecto "colaboración con otros especialistas de otras disciplinas..." , 10 asignaturas (Bioética I, Bioética II, Filosofía de los Derechos Humanos I, Filosofía de los Derechos Humanos II, Antropología Filosófica, Filosofía de la Educación, Ética I, Ética II, Axiología I, Axiología II) evidencian una ERP; mientras que en 7 asignaturas (Filosofía del Derecho, Teoría del Estado y Constitución, Religiones Comparadas, Fenomenología de la Religión, Filosofía Social, Deontología, Filosofía Política), no existe relación $(\mathrm{NER})$.

De lo dicho anteriormente, se puede inferir que el docente ha de motivar al alumno para que trascienda la actividad de interpretar y comprender textos filosóficos en abstracto y los pueda correlacionar con la vida cotidiana y, progresivamente, aprenda a trabajar de forma colaborativa e interdisciplinaria con los demás. En efecto, la Filosofía no solo sirve para explicar y describir la realidad, sino también para transformarla, desde el empoderamiento de una actitud reflexiva y comprometida con la sociedad misma.

\section{Análisis de las encuestas a docentes}

Con el objetivo de identificar las actividades y técnicas didácticas que promueven los docentes para integrar las competencias específicas de las asignaturas del área de Filosofía Práctica con el Perfil de Egreso de la Licenciatura en FEV, consultamos a los profesores que impartían dichas asignaturas y obtuvimos los siguientes resultados más significativos.

El $63 \%$ de los encuestados, manifiestan que al elaborar la programación didáctica semestral sí relacionan el perfil de egreso con las competencias específicas. Sin dudas, el planeamiento didáctico es clave para operacionalizar o visibilizar el perfil de egreso. Ahí se selecciona el contenido, se toman en cuenta las competencias específicas y se prevé de algún modo las 


\section{Anibal Beitía A.}

actividades pedagógicas que reforzarán el proceso de enseñanza-aprendizaje todo lo cual contribuye a mantener la secuencia entre los contenidos temáticos y el tipo de profesional que se desea formar.

Así mismo, se les preguntó a los profesores encuestados, qué técnicas y actividades que utilizan para potenciar en los alumnos el área de conocimiento, de habilidades y de actitudes referente al perfil de egreso

- En cuanto al área de conocimiento, el $17.95 \%$ emplea el ensayo, seguido de un $15.38 \%$ que utiliza la lectura analítica de textos filosóficos y, con igual porcentaje (12.82\%), aparecen empatados las técnicas del debate y de la exposición dialogada.

- En cuanto al área de habilidades, el $20.69 \%$ utiliza la lectura analítica de textos filosóficos y luego, con el mismo porcentaje (13.79\%), aparecen el ensayo, el debate y la exposición oral dialogada. No obstante, consideramos que las habilidades se cultivarían también cuando los estudiantes hacen algo en concreto o se aproximan a esa realidad de hacer, como podría ser el estudio de casos (que aparece con apenas un 3.45\%), entre otras técnicas didácticas.

- En cuanto al área de actitudes, el 19.23\% de los profesores encuestados considera que, a través de la lectura analítica de textos filosóficos, se podría reforzar esta área, seguido de un $15.38 \%$ que consideran que se podría cultivar a través de debates y resúmenes. Sin embargo, consideramos que el análisis de textos filosóficos, como los resúmenes, pueden fortalecer grandemente las competencias cognitivas.

A través del debate se aprende a escuchar con respeto, a tolerar las ideas con las que no se está de acuerdo. Esta parte "humana" de cultivar buenas actitudes, es fundamental que esté presente en el perfil del profesional de la Filosofía. Precisamente, es el área que los docentes encuestados consideran como la más débil en la formación actual de los estudiantes que cursan la carrera de Filosofía. 
En la Figura 4, se visualiza que, para los docentes, el área del perfil de egreso más débil en la formación actual de los estudiantes, es la de actitudes (38\%), seguida muy de cerca por el área de habilidades (37\%) y en tercer lugar el área de conocimiento (25\%).

\section{Figura 4.}

Área del perfil de egreso que los profesores consideran como la más débil en la formación actual de los estudiantes de la Licenciatura en FEV.

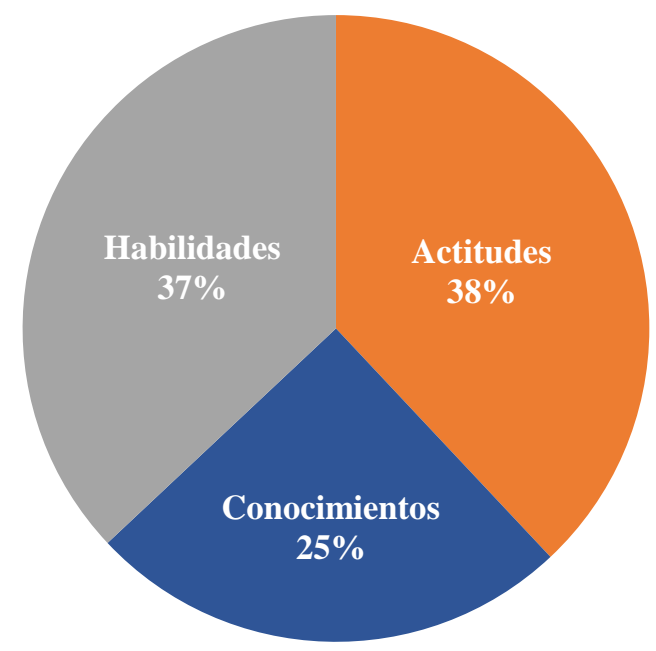

Fuente: Encuestas aplicadas a docentes de la Licenciatura en FEV, que tienen a cargo asignaturas del área de Filosofía Práctica. 2017.

Por lo tanto, se puede inferir que es necesario forjar mejores actitudes en los futuros profesionales de la Filosofía y evitar lo que se podría llamar cierta "vanidad intelectual". Recordemos que precisamente uno de los quehaceres del filósofo es generar la reflexión a través de continuas preguntas en la búsqueda de respuestas asertivas; y que toda tarea reflexiva esperaría una actitud de escucha, de diálogo fraterno y por supuesto, de un auténtico espíritu de "comunidad de aprendizaje". 


\section{Anibal Beitía A.}

\section{Análisis de las encuestas a estudiantes}

De la aplicación de las encuestas a 21 estudiantes se evidencia, de manera más significativa lo siguiente:

Respecto a si conocían las bases metodológicas para la enseñanza de la Filosofía, el 65\% señala que sí las conocen frente a un 35\% que respondió negativamente. Es esencial que los egresados de la Licenciatura en Filosofía, Ética y Valores puedan fomentar el aprender a filosofar y hacerlo con un dinamismo pedagógico. De ahí, que resulte imprescindible favorecer el conocimiento y apropiación de las bases metodológicas para la enseñanza de la Filosofía en los estudiantes que cursan en la actualidad esta carrera. Esta es precisamente una de las competencias específicas que habría que articular más con el perfil de egreso, a saber, el empoderamiento de una adecuada metodología didáctico-filosófica; que ayudaría a eliminar progresivamente el mito que la filosofía es difícil de enseñar y de comprender.

Por otra parte, a los estudiantes encuestados se les pidió responder si tenían dominio para saber interpretar y generar nueva información de carácter filosófico, a esto el 20\% manifestó que no, frente al $80 \%$ que respondió afirmativamente. La comprensión de la información que contiene un texto filosófico implica la habilidad de interpretarlo adecuadamente. Para ello, se requiere la capacidad de relacionar las partes con el todo y viceversa, comparar, secuenciar, etc.

Por lo tanto, habría que reforzar en los estudiantes universitarios de la Licenciatura en FEV, la adquisición de las competencias para interpretar y generar nueva información de carácter filosófico. Recordemos que una sólida formación profesional filosófica demanda del cultivo de competencias cognitivas para valorar con profundidad la información y construir nuevos conocimientos.

Ante la pregunta, de si durante la formación académica se desarrolla la habilidad de argumentar y refutar cuestiones filosóficas actuales; el 80\% respondió afirmativamente mientras que el 20\% dijo que no. Es importante señalar que una de las competencias propias que se requieren en el área de la Filosofía Práctica es precisamente, la habilidad para argumentar y refutar dilemas éticos actuales. En definitiva, aplicar planteamientos éticos a situaciones morales controversiales, requiere de una reflexión profunda para considerar varias opciones y así elegir la mejor en ese 
caso humano concreto.

Por consiguiente, ante la pregunta de si conocían el perfil de egreso de la Licenciatura en FEV, el $65 \%$ respondió afirmativamente y un 35\% señaló que no, tal como se aprecia en la Figura 5.

\section{Figura 5.}

Conocimiento por los estudiantes del perfil de egreso de la Licenciatura en FEV. Año 2017.

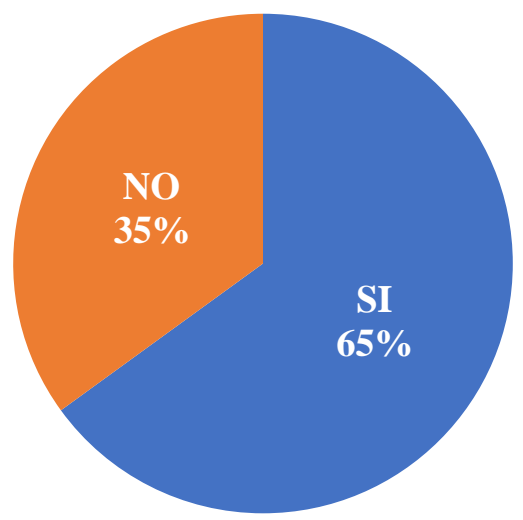

Fuente: Encuestas aplicadas a estudiantes de la Licenciatura en FEV que cursaban el I, II, III y IV año. 2017.

El perfil de egreso constituye uno de los componentes básicos del diseño curricular. Permite al estudiante universitario proyectarse o visualizarse como un profesional idóneo, con los conocimientos, habilidades y actitudes propias de su disciplina; en este caso, del quehacer filosófico. Para ello, directa o indirectamente, cada profesor fomentará que sus estudiantes se "apropien" de dicho perfil.

\section{CONCLUSIONES}

Concebir el currículum desde la perspectiva de un modelo basado en competencias, significa recuperar la importancia y validez que tienen los procesos de aprendizajes. Así, al aproximarnos a la dimensión epistemológica, cognitiva y pedagógica que tiene como fundamento subyacente 


\section{Anibal Beitía A.}

el Diseño Curricular de la Licenciatura en Humanidades con especialización en Filosofía, Ética y Valores, se devela la necesidad de articular oportunamente las competencias específicas con el perfil de egreso, desde el programa analítico de cada asignatura del área de Filosofía Práctica.

El conocimiento por sí mismo no necesariamente va a forjar sólidos profesionales. Por lo tanto, se requiere durante el proceso formativo, el fortalecimiento de actitudes éticas y axiológicas, que favorezcan un crecimiento académico y humanístico del futuro Licenciado en FEV. Esto indica que no es prudente cultivar competencias cognitivas separadas de valores éticos-sociales que han de inspirar a la construcción de una sociedad más justa, fraterna y equitativa.

Para promover una visión renovada del proceso formativo del futuro Licenciado en Filosofía, es imprescindible ofrecer una formación curricular actualizada al docente universitario que imparte clases en el Departamento de Filosofía de la Universidad de Panamá, en el área de Filosofía Práctica. Esto implica, por una parte, mejorar la calidad de los métodos y estrategias de enseñanza - aprendizaje de la Filosofía. Por otra parte, se trata de que el docente asuma, a su vez, los nuevos paradigmas educativos; es decir, que se convierta en un facilitador o mediador del aprendizaje. Para ello, irá dejando prudentemente a un segundo lugar, el modelo educativo tradicional, donde el protagonista es precisamente el docente con la famosa y válida aún “clase magistral” y así, dar paso progresivamente a una pedagogía, inspirada en el enfoque constructivista.

El nivel de apropiación de las competencias específicas que tienen actualmente los estudiantes de la Licenciatura en FEV, indica que hay que potenciarlas más. Las competencias son habilidades que se articulan continuamente y que progresivamente, conducirían hacia el saber conocer, saber ser y saber hacer de quien se dedica a la tarea filosófica. En efecto, el estudiante universitario de Filosofía debería cultivar con profundidad creciente un pensamiento críticoreflexivo y aprender a tomar decisiones asertivas en situaciones complejas de la vida cotidiana. Para saber elegir bien, es necesario saber argumentar y debatir válidamente. Una forma de desarrollar esta competencia sería creando espacios académicos interdisciplinarios. De esta forma, el docente fomentaría en los estudiantes, la posibilidad de que además de procesar la información, la puedan reconstruir desde otros enfoques y aplicarla en nuevas situaciones. 


\section{REFERENCIAS}

Cuéllar, H. (2012). ¿Qué es la filosofía de la educación?. México, Editorial Trillas.

Flamarique, L. (2008). Enseñanza de la Filosofía. Apuntes para la Universidad del siglo XXI.

$\begin{array}{lllll}\text { Pensamiento } & \text { y } \quad \text { Cultura, } & \text { 95-112. }\end{array}$ https://www.academia.edu/24448030/Enseñanza_de_la_filosofía._Apuntes_para_la_uni versidad_del_siglo_XXI

González, L. (2007). Formación Universitaria por competencias 2007-16. Centro $\begin{array}{llll}\text { Interuniversitario de } & \text { Desarrollo }\end{array}$ https://www.researchgate.net/publication/275275474_Formacion_universitaria_por_co mpetencias_2007-16

Ortiz, A. (2014). Currículo y Didáctica. Ediciones de la U, Bogotá.

Sanz de Acedo, M. (2012). Competencias cognitivas en Educación Superior. Madrid, Narcea, S.A. de Ediciones.

Santivañez, V. (2012). Diseño curricular a partir de competencias. Bogotá, Ediciones de la U.

Solange, C., Herrero M., García L. (2007). Formas de racionalidad filosófica: su impacto en la metodología de la enseñanza de la Filosofía. Cuadernos de Educación. (Número 5), pp. 159- 174. https://revistas.unc.edu.ar/index.php/Cuadernos/article/viewFile/726/686

Tobón, S. (2013). Formación integral y competencias. Pensamiento complejo, currículo, didáctica y evaluación. ECOE ediciones.

Tovar, M. y Sarmiento P. (2011). El diseño curricular, una responsabilidad compartida. http://www.redalyc.org/articulo.oa?id=28321543012

UNESCO. (2009). Enseñanza de la Filosofía en América Latina y el Caribe. Montevideo, Uruguay. Recuperado de https://unesdoc.unesco.org/ark:/48223/pf0000185119_spa

Universidad de Panamá. (2008). Modelo Educativo y Académico de la Universidad de Panamá. Ediciones Universidad de Panamá.

Universidad de Panamá. (2008). Estatuto Universitario. Ediciones Universidad de Panamá 